\title{
Study of Ultra-Thin Silicon Micropillar Based Vapor Chamber
}

\author{
Mengyao Wei ${ }^{1,2}$, Bin He ${ }^{1}$, Qian Liang ${ }^{1}$, Sivanand Somasundaram ${ }^{1}$, Chuan Seng Tan ${ }^{1,2}$, Evelyn N. Wang ${ }^{1,3}$ \\ ${ }^{1}$ Low Energy Electronic Systems (LEES) IRG, Singapore-MIT Alliance for Research and Technology (SMART) \\ Centre, 1 CREATE Way, Singapore 138602 \\ ${ }^{2}$ School of Electrical and Electronics Engineering, Nanyang Technological University \\ 50 Nanyang Avenue, Singapore 639798 \\ ${ }^{3}$ Department of Mechanical Engineering, Massachusetts Institute of Technology \\ 77 Massachusetts Avenue, Cambridge, MA 02139, United States \\ enwang@mit.edu
}

\begin{abstract}
Silicon vapor chamber has drawn much attention in recent years, owing to its mature microfabrication process, accurate control over its geometries, direct integration with semiconductor devices that can eliminate the thermal interface resistance and prevent thermal expansion mismatch. In this paper, micropillar wicks that can generate excellent capillary performance and possess high permeability was adopted as the evaporator and adiabatic wick structure. Ultra-thin vapor chambers with thickness of only 1.25 $\mathrm{mm}$ were fabricated, the total size of the vapor chamber was $4 \mathrm{~cm} \times 4 \mathrm{~cm} \times 1.25 \mathrm{~mm}$. As essential parts of a vapor chamber, the geometric sizes of the micropillars at evaporator and adiabatic regions were optimized. Based on the Brinkman equation derived dryout heat flux model, the optimal geometric combinations for evaporator and adiabatic regions were $d=17.4 \mu \mathrm{m}, h=l=30.6 \mu \mathrm{m}$ and $d=15$ $\mu \mathrm{m}, h=l=30.6 \mu \mathrm{m}$ respectively. Actual sizes after micro fabrication was $d=18.9 \mu \mathrm{m}, h=31.3 \mu \mathrm{m}, l=30.6 \mu \mathrm{m}$ and $d=15.8 \mu \mathrm{m}, h$ $=31.3 \mu \mathrm{m}, l=30.6 \mu \mathrm{m}$ for evaporator and adiabatic regions respectively. Performance comparison between optimized and nonoptimized samples has shown that the optimized sample performed best, which can dissipate a high heat flux of $98.1 \mathrm{~W} / \mathrm{cm}^{2}$ before dryout. The deviation between model predicted and experimentally measured dryout heat flux was only $11.2 \%$, which validated the model with high accuracy. Effective thermal resistance of various samples was also studied. A smallest effective thermal resistance of $0.53 \mathrm{~K} / \mathrm{W}$ can be obtained. Effective thermal resistance was found to decrease with heat flux before dryout while a reverse trend was observed after dryout. The vapor chamber was also found to have a good temperature uniformity. The largest temperature difference was only $9.6{ }^{\circ} \mathrm{C}$ at very high heat load of $98.1 \mathrm{~W}$. This paper demonstrated significant insights into the investigation of silicon vapor chamber, and can be used as useful design guidance for micropillar based vapor chambers.
\end{abstract}

Keywords: vapor chamber, micropillar, dryout heat flux, optimization

\section{Introduction}

High density electronic devices are facing challenges of generating large amount of concentrated heat, which severely limit their performance and reliability. Vapor chamber, which is a 2D flat heat pipe, has the capability of dissipating heat effectively and passively with good temperature uniformity. When heat is applied to the evaporator section of a vapor chamber, working fluid enclosed inside the chamber is evaporated and became vapor. Due to the pressure difference between the hot evaporator part and the cold condenser part, the vapor flows to the condenser and condensed back into liquid droplets. The liquid was then transported back to the evaporator via the capillary pressure developed in the porous adiabatic and evaporator regions. Conventional vapor chambers was made of highly conductive metals like copper and aluminium [1-4]. Metal vapor chambers require thermal interface layer to be attached to the integrated electronic systems, which introduce extra thermal interface resistance. Due to the thermal expansion coefficient difference between metal vapor chambers and semiconductor devices, thermal expansion mismatch is inevitable. Thus, silicon vapor chambers were widely studied in recent years [5-11]. Vapor chambers made of silicon possess advantages of direct integration with semiconductor devices, elimination of potential thermal expansion mismatch, relatively high thermal conductivity of 150 $\mathrm{W} / \mathrm{m} \cdot \mathrm{K}$ of silicon and can be fabricated using the mature microelectronic manufacturing process.

Vadakkan et. al. [6] constructed a numerical thermal model and conducted finite element analysis for silicon vapor chamber with water as working fluid. They found that silicon vapor chamber can have equal or even better performance 
than a copper vapor chamber. Compressive stress in the silicon die had $96 \%$ reduction by substituting copper vapor chamber with a silicon one. This is due to the elimination of thermal expansion mismatch problem and high compliance of silicon. Cai et al. [9, 10] developed a silicon vapor chamber with a triple-stack structure. Glass frit bonding was performed to seal the vapor chamber. Mechanical optimization simulation was done to determine the position of supporting posts. For a $3 \mathrm{~mm} \times 38 \mathrm{~mm} \times 38 \mathrm{~mm}$ silicon vapor chamber prototype, effective thermal conductivity of $2500 \mathrm{~W} / \mathrm{m} \cdot \mathrm{K}$ was obtained. The device was also proven to have good mechanical strength and was hermetically sealed. They also investigated the material and process compatibility of the silicon vapor chamber[5]. Reliable silicon vapor chambers can be obtained with purified working fluid, lead-based solder material, high glazing temperature in glass frit bonding and oxidization of silicon surface. Later on, Cai et. al. [11] fabricated an ultra-thin silicon vapor chamber with size of $50 \mathrm{~mm} \times 70 \mathrm{~mm} \times 1 \mathrm{~mm}$. The vapor chamber has $13 \times 8$ supporting pillar arrays. The evaporator was made of coarse and fine wicks. The fine wick composed of micropillars with $10 \mu \mathrm{m}$ diameter and cavities with $100 \mu \mathrm{m}$ for vapor ventilation. A high effective thermal conductivity of over $10000 \mathrm{~W} / \mathrm{m} \cdot \mathrm{K}$ was demonstrated in both $1 \mathrm{D}$ and $2 \mathrm{D}$ heat transfer modes.

Among various sections of the vapor chamber, evaporator plays a significant role as it circulates the working fluid and possess the largest thermal resistance. Thus to demonstrate a high performance silicon vapor chamber, the optimization of evaporator is essential. Control over the fabrication process and constructing model for conventional wire mesh, sintered powder and microgroove evaporator wicks were difficult. Micropillar evaporator has the advantage of accurate control over the fabrication process, high liquid transport capability [12-15] and was widely studied. Although a few of foregoing researches were done on silicon vapor chambers. The selection of evaporator geometries was trial-and-error based. In this paper, the optimal geometric combination was obtained based on a theoretical model that predicted dryout heat flux of the evaporator. Ultra-thin silicon vapor chambers were fabricated using standard micro manufacture process and sealed by eutectic bonding. Thermal tests were performed to examine the heat dissipation capability and temperature uniformity of the vapor chamber.

\section{Model and Optimization}

Based on Wang et. al.'s [16] work, the Darcy's law based dryout heat flux for 2D micropillar arrays was expressed as:

$$
q^{\prime \prime}=\frac{\rho_{l} K h h_{f g} P_{c a p}}{\mu}\left[\frac{a^{2}}{2}-\frac{2}{a} \sum_{m=1}^{\infty} \frac{\sin \left(\beta_{m} a\right)}{\beta_{m}^{3} \cosh \left(\beta_{m} b\right)}\right]^{-1}
$$

where $\rho_{l}, h_{f g}$ and $\mu$ are liquid density, latent heat and viscosity respectively, which are physical properties of working fluid. $a$ and $b$ are length of studied porous medium in $x$ and $y$ directions respectively. $h$ is the height of micropillars. Factor $\beta_{m}=\frac{(2 m-1) \pi}{2 a} \cdot \varepsilon$ is the porosity of micropillar wick. For the $2 \mathrm{D}$ case where $a=b=L$,

$$
\left.\frac{a^{2}}{2}-\frac{2}{a} \sum_{m=1}^{\infty} \frac{\sin \left(\beta_{m} a\right)}{\beta_{m}^{3} \cosh \left(\beta_{m} a\right)}\right]=\frac{a^{2}}{2}-0.2053 a^{2} \approx 0.3 a^{2}=0.3 L^{2}
$$

By substituting Equation (2) into Equation (1) and extend the solution to Brinkman equation based heat flux according to $[13,15,17]$, the dryout heat flux can be expressed as:

$$
q_{\max }^{\prime \prime}=\frac{3.33 \rho_{l} K h h_{f g} P_{c a p}}{\mu L^{2}}\left[1-\frac{\tanh \left(\sqrt{\frac{\varepsilon}{K}} h\right)}{\sqrt{\frac{\varepsilon}{K}} h}\right]
$$


In Equation (3), parameters $P_{c a p}$ and $K$ represent the capillary pressure and permeability of the wick respectively. Expressions of $P_{c a p}$ and $K$ can be found in [18] and [19] respectively. Both $P_{c a p}$ and $K$ are geometric dependent and change with micropillar geometries in opposite trends. Therefore, optimal geometries that can result in largest dryout heat flux exist can be obtained through optimization in Matlab. Since temperature rise is an important performance indicator of evaporator, superheat, which is defined as the temperature difference between evaporator and ambient, was adopted in the optimization process and correlated with heat flux by the equation $q "=k_{\text {eff }} \frac{\Delta T}{h}$, where the effective thermal conductivity $k_{\text {eff }}=6.02633\left(\frac{d}{l}\right)^{2}-3.19508 \frac{d}{l}+2.12324$ [12], $d$ and $l$ are the diameter and center - to - center distance of micropillars. The optimization results are plotted in Fig. 1. To fabricate the sample and conduct parametric studies, the evaporator micropillar geometric combination at $\Delta T=15^{\circ} \mathrm{C}$ was selected, which correspond to $d=17.4 \mu \mathrm{m}, h=l=30.6 \mu \mathrm{m}$. The adiabatic region served the function of circulating liquid only, thus the optimization of micropillars in adiabatic regions only considered the fluid transportation capability, which is governed by Washburn's equation[20]. According to [20], the factor $\sqrt{K P_{\text {cap }} / \varepsilon}$ need to be optimized, while $\varepsilon=1-\pi d^{2} / 4 l^{2}$ is porosity of the micropillar wick . Height of the adiabatic region micropillars was predefined by the micropillar depth in evaporator regions, which was determined by deep reactive ion etching microfabrication process. Thus with $h=30.6 \mu \mathrm{m}$, the optimized $d=15 \mu \mathrm{m}$ and $l=30.6 \mu \mathrm{m}$. The adiabatic region has larger porosity compared to the evaporator region, which has a lower flow resistance for liquid propagation.

(a)

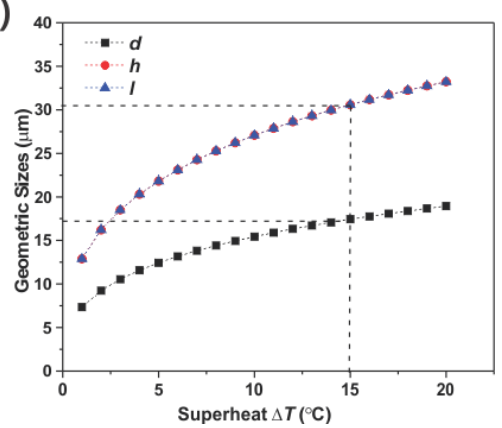

(b)

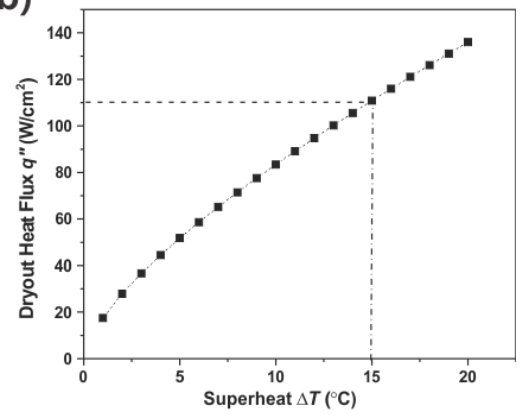

Fig. 1: Optimization results for 2D evaporator in vapor chamber (a) micropillar geometries d, h, 1 at different superheat (b) the corresponding heat flux.

\section{Experiment}

\subsection{Sample}

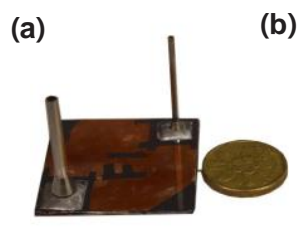

(b)

(c)
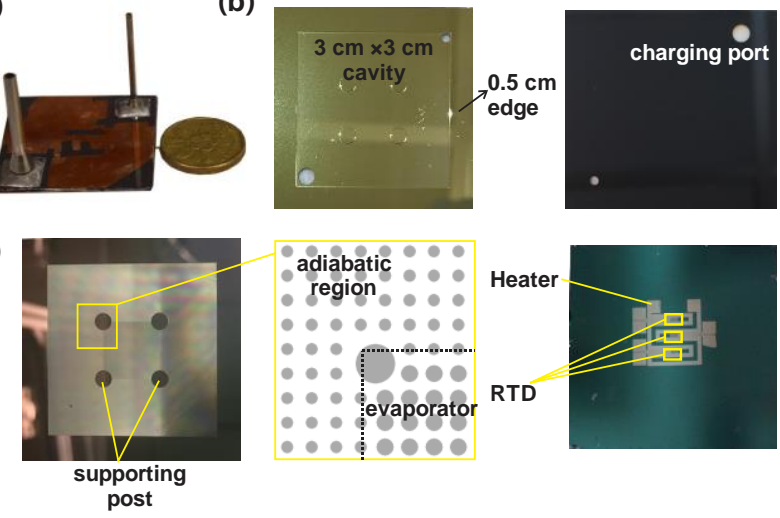

Fig. 2: (a) Sealed silicon vapor chamber with thickness of $1.25 \mathrm{~mm}$ (b) Top wafer of silicon vapor chamber with $3 \mathrm{~cm} \times 3 \mathrm{~cm} \times 100 \mu \mathrm{m}$ cavity, one charging port and one evacuation port (c) Bottom wafer with micropillar wicks on one side and $1 \mathrm{~cm} \times 1 \mathrm{~cm} P t$ heater and RTDs on the other side. Geometries for adiabatic and evaporator regions were optimized. 
Detailed fabrication process was described in our previous work [7]. The ultra-thin silicon vapor chamber was made up of two silicon wafers that were $\mathrm{Au}-\mathrm{Si}$ eutectic bonded together at $400^{\circ} \mathrm{C}$. Both the top and bottom wafers were $4 \mathrm{~cm} \times$ $4 \mathrm{~cm}$ in size, with $0.5 \mathrm{~cm}$ edges for bonding purpose. The top wafer, which was act as the condenser, has a $100 \mu \mathrm{m}$ deep reactive ion etched (DRIE) cavity of $3 \mathrm{~cm} \times 3 \mathrm{~cm}$ as the vapor space for the vapor chamber, as shown in Fig. 2(b). Backside of the top wafer has $150 \mathrm{~nm} \mu \mathrm{m}$ and $500 \mu \mathrm{m}$ Au for eutectic bonding. There are two holes with size of 1/8" and $1 / 16$ " to connect to two tubes. The $1 / 16$ ' tube was used for evacuating the vapor chamber to vacuum while working fluid was charged to the vapor chamber through the 1/8" tube. $100 \mathrm{Ti}$ and $500 \mathrm{~nm} \mathrm{Cu}$ were deposited on the front side of the top wafer, this was to create soldering pads for the tubes. The bottom wafer has photolithography defined micropillar wicks on one side, and $1 \mathrm{~cm} \times 1 \mathrm{~cm}$ size $150 \mathrm{~nm}$ thick Pt heater and resistance temperature detector (RTDs) on the other side. There was a SiO2 layer grown by thermal oxidization with thickness of $900 \mu \mathrm{m}$ in between of the Pt films and the wafer. Size of the evaporator was $1 \mathrm{~cm} \times 1 \mathrm{~cm}$, it was located at the center of the micropillar wick. The micropillar wick included the evaporator and the adiabatic regions and has a total size of $3 \mathrm{~cm} \times 3 \mathrm{~cm}$, as shown in Fig. 2(c). Height of micropillar was etched by DRIE. There were 4 supporting posts with diameter of $3 \mathrm{~mm}$ at 4 corners of the evaporator to enhance the mechanical strength of the vapor chamber. Total thickness of the vapor chamber was only $1.25 \mathrm{~mm}$, which was thinner than a 1 dollar Singapore coin Fig. 2(a). The ultra-small thickness and light weight of this vapor chamber made it to be an excellent candidate for compact electronic devices. Samples with different geometries are listed in Table 1. Sample 2 was designed based on optimized evaporator and adiabatic wick geometries. Actual size after microfabrication was measured by SEM and listed in Table 1.

Table 1: List of Samples.

\begin{tabular}{|c|c|c|c|c|c|c|}
\hline \multirow{2}{*}{$\begin{array}{c}\text { Sample } \\
\#\end{array}$} & \multicolumn{3}{|c|}{ Evaporator } & \multicolumn{3}{c|}{ Adiabatic Region } \\
\cline { 2 - 7 } & $\boldsymbol{d}(\boldsymbol{\mu \mathbf { m }})$ & $\boldsymbol{h}(\boldsymbol{\mu \mathbf { m }})$ & $\boldsymbol{l}(\boldsymbol{\mu \mathbf { m }})$ & $\boldsymbol{d}(\boldsymbol{\mu \mathbf { m }})$ & $\boldsymbol{h}(\boldsymbol{\mu \mathbf { m }})$ & $\boldsymbol{l}(\boldsymbol{\mu \mathbf { m }})$ \\
\hline $\mathbf{1}$ & 15.5 & 31.3 & 30.6 & 15.8 & 31.3 & 30.6 \\
\hline $\mathbf{2}$ & 18.9 & 31.3 & 30.6 & 15.8 & 31.3 & 30.6 \\
\hline $\mathbf{3}$ & 15.5 & 31.3 & 27 & 15.8 & 31.3 & 30.6 \\
\hline $\mathbf{4}$ & 4.6 & 17.7 & 16 & 7.8 & 17.7 & 16 \\
\hline $\mathbf{5}$ & 9.2 & 17.7 & 16 & 7.8 & 17.7 & 16 \\
\hline
\end{tabular}

\subsection{Experimental Setup}

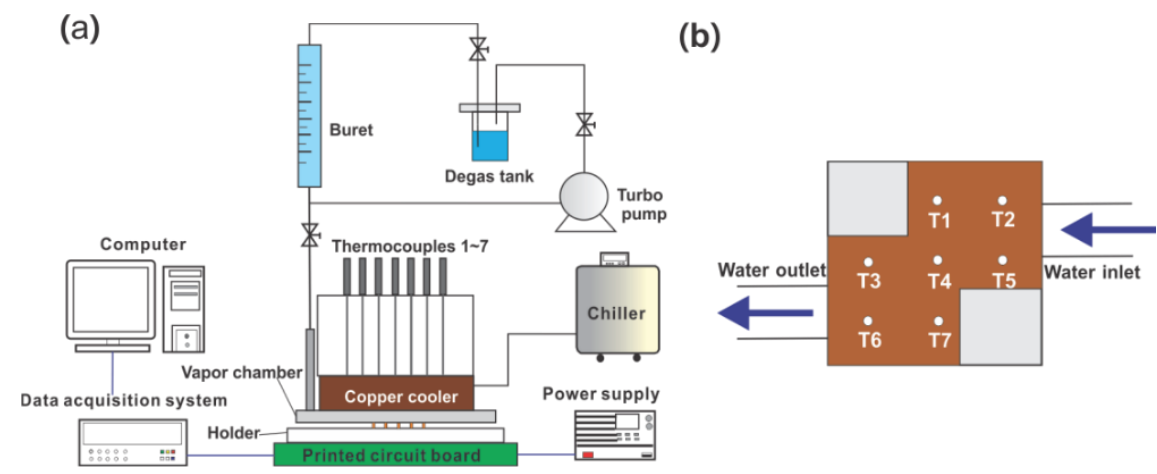

Fig. 3: (a) Schematic illustration of experimental setup (not in scale): the silicon vapor chamber was mounted onto a printed circuit board which was connected to the power supply and data acquisition system. Vapor chamber was water cooled by a copper condenser. Temperature of the condenser was measured by 7 thermocouples. (b) Location of 7 thermocouples.

The experimental setup is schematically illustrated in Fig. 3(a). Prior to thermal characterization, the vapor chamber samples were evacuated to a vacuum level of $10^{-4}$ mbar to remove any non-condensable gas inside. During the evacuation process, the samples were monitored by a Pirani gauge (Leyold Thermovac TTR 91N) to ensure that there were no leakage of gas from the samples. Then the vapor chambers were charged with degassed DI water. Degassing was done through 3 freeze-pump-thaw cycles, details can be found in $[13,15]$. The degassed DI water was then filled in to a $3 \mathrm{ml}$ burette with accuracy of $10 \mu \mathrm{l}$ in order to control the liquid amount charged into the sample 
accurately. Desired charging amount was calculated based on the geometries of the bottom wicks, to ensure that all the space in between of micropillars were occupied by working fluid and there was no flooding of the wick. A copper cooler that was water cooled by a chiller with temperature of $15^{\circ} \mathrm{C}$, was in contact with the vapor chamber as heat sink. The vapor chamber was mounted onto a sample holder and connected to printed circuit board (PCB) with pogo-pins. During the tests, Pt heater on the bottom of the vapor chamber was subject to an incremental voltage heating. In the meanwhile, resistances of the Pt resistance-temperature-detectors (RTDs) were measured and recorded by the data acquisition system (DAQ, Keysight 34970a). The voltage was increased from $0 \mathrm{~V}$ to $140 \mathrm{~V}$, resistance readings were taken 4 mins after each voltage was applied, to ensure the vapor chamber has reached a steady temperature. Once the test was finished, the RTDs were calibrated against a high-precision Pt 100 thermometer (Omega) from $30^{\circ} \mathrm{C}$ to $80^{\circ} \mathrm{C}$ in an oven (OMH60, Heratherm). The resistance readings at different temperatures were averaged and the linear correlations between the resistance and temperature were obtained for each RTD. Evaporator temperature $T_{\text {eva }}$ of the vapor chamber during the tests can be calculated based on the linear correlations. Temperature of the condenser side $T_{\text {cond }}$ of the vapor chamber was measured by 7 thermocouples (Omega, TJ160-CPSS-040U-2). The distribution of 7 thermocouples was illustrated in Fig. 3(b) with T4 located at the center.

\section{Results and Discussion}

The geometries of sample 1 5 are listed in Table 1. Sample 2 has the optimized geometries according to the optimization results in section 2. Performance comparison between sample 2 and sample 1, 3, 4, 5 is plotted in Fig. 4, while micropillars of sample 1,3, 4, 5 have different geometry combinations and were not optimized. Since dryout cannot be observed from a sealed silicon vapor chamber, the turning point that indicated a change of $q$ " vs. $\Delta T$ slope was defined as the dryout heat flux, as shown in Fig. 4 by the arrows. This was due to the reduction of available thin film evaporation area after dryout, that caused an abrupt increase of temperature compared with the working regimes with thin film evaporation [13] phase change. From Fig. 4, it can be observed that, the non-optimized sample $3 \sim 5$ have dryout heat flux of less than $20 \mathrm{~W} / \mathrm{cm}^{2}$, sample 1 has dryout heat flux of around $35 \mathrm{~W} / \mathrm{cm}^{2}$, while that of optimized sample 2 was 98.1 $\mathrm{W} / \mathrm{cm}^{2}$. The optimization of micropillar geometries enhanced the performance of vapor chambers by $2.8 \sim 5$ times. This was attributed to a counterbalance between the capillary performance and permeability of the optimized sample 2 in comparison with sample 1 and 3. Compared with sample 4 and 5, a higher micropillar height of $31.3 \mu \mathrm{m}$ corresponded to a larger volumetric flow rate of liquid, which can effectively compensate the liquid evaporated and delayed the dryout. Dryout heat flux sample 2 was $110.5 \mathrm{~W} / \mathrm{cm}^{2}$ as predicted by the model. The discrepancy between model prediction and experimental measure dryout heat flux was only $11.2 \%$, which indicates the accuracy of our model.

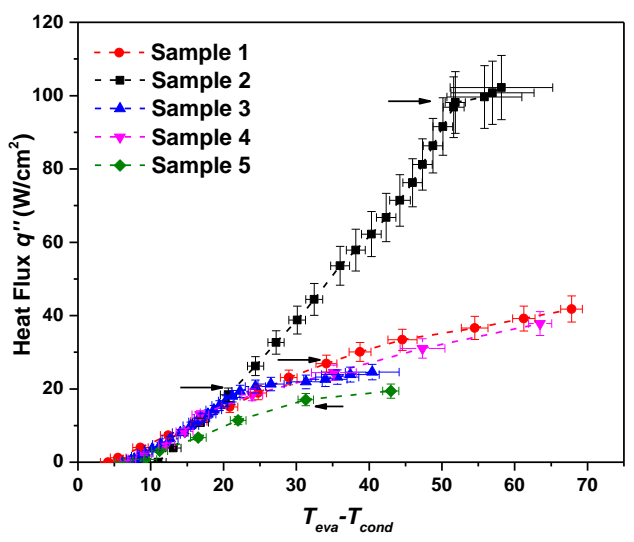

Fig. 4: Performance comparison between sample 2 (optimized), sample 1, $3 \sim 5$ (non-optimized): dryout heat flux was indicated by the arrows. Sample 2 has highest dryout heat flux of $98.1 \mathrm{~W} / \mathrm{cm}^{2}$.

Effective thermal resistance vs. heat flux $q$ "' of each sample are plotted in Fig. 5. The effective thermal resistance was calculated based on $R=\frac{T_{e v a}-T_{\text {cond }}}{q^{\prime \prime} A}$, where $A$ is heater area. According to Fig. 5, effective thermal resistance decrease 
with heat flux before the inception of dryout. This was due to the change of liquid meniscus curvature. As heat flux increased, the evaporation rate became larger and liquid meniscus receded into the space between micropillars [21], which caused the decrease of liquid film thickness and resulted in smaller effective thermal resistance. After dryout has been occurred, less micropillar wick areas were covered by liquid and the thin film evaporation area was largely reduced. Thus an increase of effective thermal resistance can be observed after dryout point has been reached. A lowest effective thermal resistance of $0.53 \mathrm{~K} / \mathrm{W}$ can be obtained for sample 2 with optimized geometries.

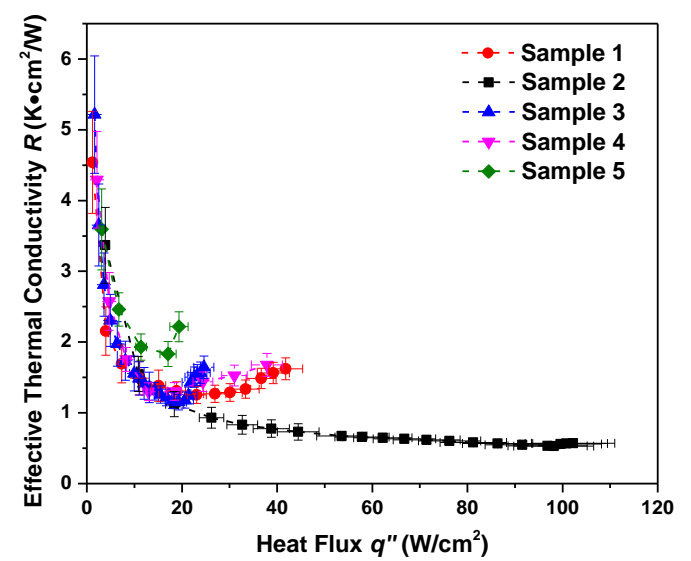

Fig. 5: Effective thermal resistance of sample $1 \sim 5$.

Fig. 6 illustrated the temperature distribution at the top of sample 2 vapor chamber at various heat loads from $10.9 \mathrm{~W}$ to $98.1 \mathrm{~W}$. From Fig. 6, it can be observed that the temperature distribution was uniform in general. The largest temperature difference was $9.6^{\circ} \mathrm{C}$ between $\mathrm{T} 4$ and $\mathrm{T} 5$ at $98.1 \mathrm{~W}$. T4 always has the highest reading as it was located at the center of the vapor chamber, while the center was heated by the Pt heater directly. Moreover, part of the total heat load has been conducted through the 4 supporting posts to the top condenser, which contributed to the high temperature T4 at the center as well. At $98.1 \mathrm{~W}$, the dryout sample 2 started to dry as shown in Fig. 4. Temperature reading of T3, T7 was larger than that of T2, T5. The reason for this temperature difference was due to the non-uniformity of the water cooling system. Lowest temperature readings was obtained at T2, T5 as it was the inlet of water to the copper cooler as shown in Fig. 3(b), at which the water has the lowest temperature. When the water flowed from the inlet to the outlet of the copper cooler, which was from T2, T5 to T3, T7, water absorbed heat and the temperature was higher at the outlet. According to [3], when the temperature difference between the water and vapor chamber was larger, more heat can be taken away. Thus the temperatures at T3 and T7 were larger. As shown in Fig. 3(b), not the entire condenser surface was in contact with the copper chiller, as extra space need to be left for the charging and evacuating tubes. This reduced the effectiveness of the copper chiller and resulted in a larger temperature difference between different thermocouples. If the copper condenser can cover the entire condenser, the temperature uniformity of the vapor chamber can be even better.

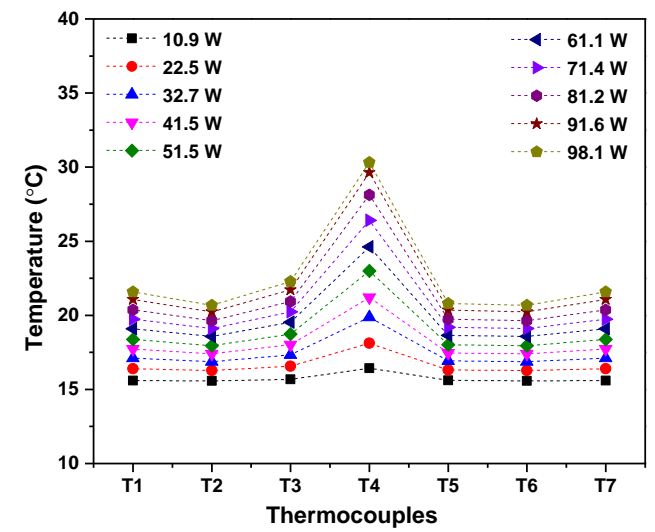

Fig. 6: Condenser temperature distribution of sample 2 at different heat loads. 


\section{Conclusion}

This paper presented a systematic study of ultra-thin micropillar based silicon vapor chamber with a thickness of only $1.25 \mathrm{~mm}$. The model that can predict the dryout heat flux, optimization of evaporator and adiabatic geometries, microfabrication and thermal characterization of silicon vapor chambers were demonstrated. The silicon vapor chambers have a total size of $4 \mathrm{~cm} \times 4 \mathrm{~cm} \times 1.25 \mathrm{~mm}$, while the wick region was $3 \mathrm{~cm} \times 3 \mathrm{~cm}$ in size with a $1 \mathrm{~cm} \times 1 \mathrm{~cm}$ evaporator. The vapor core thickness was $100 \mu \mathrm{m}$ deep and the vapor chamber was sealed by Au-Si eutectic bonding at $400{ }^{\circ} \mathrm{C}$. Vapor chambers with different micropillar geometries were fabricated and compared. Sample 2 with optimized evaporator micropillar geometries of $d=18.9 \mu \mathrm{m}, h=31.3 \mu \mathrm{m}, l=30.6 \mu \mathrm{m}$ (actual sizes) and optimized adiabatic micropillars of $d$ $=15.8 \mu \mathrm{m}, h=31.3 \mu \mathrm{m}, l=30.6 \mu \mathrm{m}$ was found to performance best. A highest dryout heat flux of $98.1 \mathrm{~W} / \mathrm{cm}^{2} \mathrm{can} \mathrm{be}$ obtained with sample 2, which only has $11.2 \%$ discrepancy with the model predicted dryout heat flux. Effective thermal resistances of various samples were investigated, it was found that effective thermal resistance decrease with heat flux before dryout while increased after dryout was occurred. This can be explained by the receding of meniscus into micropillar pores as heat flux increases, which reduced the liquid film thickness and resulted in smaller effective thermal resistance. After dryout, thin film evaporation area decreased and the effective thermal resistance increased. The vapor chamber was found to have good temperature uniformity at different heat loads. The highest temperature difference was $9.6^{\circ} \mathrm{C}$ at $98.1 \mathrm{~W}$. If a condenser that can cover the entire top surface of the vapor chamber can be adopted, the vapor chamber has the potential to possess better temperature uniformity. In conclusion, this paper provided great insights into the study of ultra-thin silicon vapor chamber. The optimization results can also be used for the design of micropillar based vapor chamber with different design requirements.

\section{Acknowledgements}

Funding support from National Research Foundation Singapore and Singapore-MIT Alliance for Research and Technology's Low Energy Electronic Systems (LEES) IRG are gratefully acknowledged.

\section{References}

[1] H. Peng, J. Li, and X. Ling, "Study on heat transfer performance of an aluminum flat plate heat pipe with fins in vapor chamber," Energy Conversion and Management, vol. 74, pp. 44-50, 2013.

[2] S.-C. Wong, K.-C. Hsieh, J.-D. Wu, and W.-L. Han, "A novel vapor chamber and its performance," International Journal of Heat and Mass Transfer, vol. 53, pp. 2377-2384, 2010.

[3] Y. Tang, D. Yuan, L. Lu, and Z. Wang, "A multi-artery vapor chamber and its performance," Applied Thermal Engineering, vol. 60, pp. 15-23, 2013.

[4] X. Ji, J. Xu, and A. M. Abanda, "Copper foam based vapor chamber for high heat flux dissipation," Experimental Thermal and Fluid Science, vol. 40, pp. 93-102, 2012.

[5] C. Qingjun, B. Avijit, T. Chialun, W. K. Martin, and F. D. Jeffrey, "Studies of material and process compatibility in developing compact silicon vapor chambers," Journal of Micromechanics and Microengineering, vol. 23, p. 065003, 2013.

[6] U. Vadakkan, G. M. Chrysler, and S. Sane, "Silicon/water vapor chamber as heat spreaders for microelectronic packages," in Semiconductor Thermal Measurement and Management Symposium, 2005 IEEE Twenty First Annual IEEE, pp. 182-186, 2005.

[7] B. He, M. Wei, S. Somasundaram, C. S. Tan, and E. N. Wang, "Experiments on the ultrathin silicon vapor chamber for enhanced heat transfer performance," in 2016 15th IEEE Intersociety Conference on Thermal and Thermomechanical Phenomena in Electronic Systems (ITherm), pp. 569-573, 2016.

[8] C. Gillot, A. Laï, M. Ivanova, Y. Avenas, C. Schaeffer, and E. Fournier, "Experimental study of a flat silicon heat pipe with microcapillary grooves," in InterSociety Conference on Thermal and Thermomechanical Phenomena in Electronic Systems, 2004.

[9] Q. Cai, B.-c. Chen, C. Tsai, Y. Zhao, and C.-1. Chen, "Development of Silicon Based Heat Spreader for High Power Electronic Devices," in ASME 2009 Second International Conference on Micro/Nanoscale Heat and Mass Transfer, 2009, pp. 443-448.

[10] Q. Cai, B.-c. Chen, and C. Tsai, "Design, development and tests of high-performance silicon vapor chamber," Journal of Micromechanics and Microengineering, vol. 22, p. 035009, 2012. 
[11] S. Q. Cai, Y.-C. Chen, and A. Bhunia, "Design, development and tests of a compact thermofluid system," Applied Thermal Engineering, vol. 102, pp. 1320-1327, 2016.

[12] M. Wei, S. Somasundaram, B. He, Q. Liang, R. Raj, C. S. Tan, et al., "Optimization of Biporous Micropillar Array for Enhanced Heat Transfer Performance," in ASME 2015 International Mechanical Engineering Congress and Exposition, pp. V08BT10A002-V08BT10A002, 2015.

[13] M. Wei, B. He, S. Somasundaram, C. S. Tan, and E. N. Wang, "Optimization and thermal characterization of uniform micropillar based silicon evaporator in advanced vapor chambers," in 2016 15th IEEE Intersociety Conference on Thermal and Thermomechanical Phenomena in Electronic Systems (ITherm), pp. 1019-1023, 2016.

[14] B. He, M. Wei, Q. Liang, C. S. Tan, and E. N. Wang, "Experiments on the Biporous Micropillar Array for Enhanced Heat Transfer Performance," in ASME 2016 5th International Conference on Micro/Nanoscale Heat and Mass Transfer, pp. V002T12A001-V002T12A001, 2016.

[15] M. Wei, S. Somasundaram, B. He, Q. Liang, C. S. Tan, and E. N. Wang, "Experimental characterization of Si micropillar based evaporator for advanced vapor chambers," in Electronics Packaging Technology Conference (EPTC), 2014 IEEE 16th, pp. 335-340, 2014.

[16] Y. Wang and G. Peterson, "Analytical model for capillary evaporation limitation in thin porous layers," Journal of thermophysics and heat transfer, vol. 17, pp. 145-149, 2003.

[17] L. Qian, R. Raj, S. Adera, S. Somasundaram, T. Chuan Seng, and E. N. Wang, "Experiment and modeling of microstructured capillary wicks for thermal management of electronics," in Electronics Packaging Technology Conference (EPTC 2013), 2013 IEEE 15th, pp. 592-597, 2013.

[18] R. Xiao, R. Enright, and E. N. Wang, "Prediction and optimization of liquid propagation in micropillar arrays," Langmuir, vol. 26, pp. 15070-15075, 2010.

[19] C. Byon and S. J. Kim, "The effect of meniscus on the permeability of micro-post arrays," Journal of Micromechanics and Microengineering, vol. 21, p. 115011, 2011.

[20] E. W. Washburn, "The dynamics of capillary flow," Physical review, vol. 17, p. 273, 1921.

[21] D. Ćoso, V. Srinivasan, M.-C. Lu, J.-Y. Chang, and A. Majumdar, "Enhanced heat transfer in biporous wicks in the thin liquid film evaporation and boiling regimes," Journal of Heat Transfer, vol. 134, p. 101501, 2012. 